\title{
Vall 09Asp Polymorphism of the Omentin-I Gene and Incidence of Knee Osteoarthritis in a Chinese Han Population: A Correlation Analysis
}

\author{
Ruofei Chen',* \\ Yaqin Zhang ${ }^{\mathrm{I}} *$ \\ Honggang $\mathrm{Xu}^{2}$ \\ Huaqing $\mathrm{Hu}^{3}$ \\ Mingwei Chen (iD ${ }^{4}$ \\ Zongwen Shuai'
}

'Department of Rheumatology and Immunology, The First Affiliated Hospital of Anhui Medical University, Hefei, 230032, Anhui Province, People's

Republic of China; ${ }^{2}$ Department of Sports Injury and Arthroscopic Surgery,

The First Affiliated Hospital of Anhui Medical University, Hefei, 230032, Anhui Province, People's Republic of China; ${ }^{3}$ Health Management Center, The First Affiliated Hospital of Anhui Medical University, Hefei, 230032, Anhui

Province, People's Republic of China; ${ }^{4}$ Department of Endocrinology, The First Affiliated Hospital of Anhui Medical University, Hefei, 230032, Anhui Province, People's Republic of China

*These authors contributed equally to this work

\begin{abstract}
Objective: To investigate the correlation of the Val109Asp polymorphism of the omentin-1 gene with the risk and severity of knee osteoarthritis (KOA) in a Chinese Han population. Methods: This study enrolled 383 patients with primary KOA and 460 healthy controls. The genotypes were determined by the detection of single nucleotide polymorphism. To explore the interaction between omentin-1 gene polymorphism and obesity and age, the body mass index (BMI) of $25 \mathrm{~kg} / \mathrm{m}^{2}$ and the age of 55 years old were preset as the cut-off value of stratified analysis. Furthermore, enzyme-linked immunosorbent assay was used to determine the levels of omentin-1, interleukin (IL)-1 $\beta$, IL-6 in peripheral blood and synovial fluid and the contents of IL-1 $\beta$, IL-6, metalloproteinase (MMP)-13 and collagen (COL)-II in the supernatant of knee joint cartilage tissue.
\end{abstract}

Results: The Val109Asp polymorphism of the omentin-1 gene showed no obvious correlation with KOA. Compared with Asp/Asp genotype carriers with BMI $<25 \mathrm{~kg} / \mathrm{m}^{2}$ and age $<55$ years old, Val109 allele carriers with $\mathrm{BMI} \geq 25 \mathrm{~kg} / \mathrm{m}^{2}$ and age $\geq 55$ years old had obviously increased risk of KOA (adjusted $\mathrm{OR}=1.416, p=0.042$; adjusted $\mathrm{OR}=1.735, p=0.038$, respectively). In the KOA group, only the omentin-1 levels were significantly lower in the plasma and synovial fluid of Ala/Ala genotype carriers than in those of Asp/Asp genotype carriers. Meanwhile, the proportion of patients with moderate-severe K-L Classification, the levels of IL-1 $\beta$, IL-6 in synovial fluid and the expression levels of IL-1 $\beta$, IL-6 and MMP-13 in cartilage tissue significantly increased $(p<0.05)$. By contrast, the expression level of COL-II in cartilage tissue significantly decreased $(p<0.05)$.

Conclusions: The Val109Asp polymorphism of the omentin-1 gene may not be the primary pathogenic factor of KOA in Chinese. The Val/Val genotype can be regarded as a potential biomarker for the risk of KOA progression.

Keywords: omentin-1, single nucleotide polymorphism, knee osteoarthritis, biomarker, Chinese, obesity

\section{Background}

Osteoarthritis (OA), characterised by impairment in the normal synthesis and degradation of articular cartilage, extracellular matrix and subchondral bone and synovial inflammation and synovial fibrosis as well as inflammation and fibrosis of the infrapatellar fat pad $^{1-3}$ is a prevalent degenerative joint disorder caused by mechanical and biological factors. It is the leading cause of pain and disability and the primary factor for the decline of patients' quality of life. ${ }^{4}$ Knee osteoarthritis (KOA) is the most common form of OA, which can bring intolerable pain to
Correspondence: Mingwei Chen;

Zongwen Shuai

Email chmwl@163.com;

amushuaizw@163.com 
patients and requires frequent surgical treatment. At present, it is well known that gender, age, trauma, joint injury, excessive physical activity, obesity, and other factors are the risk factors of KOA. ${ }^{5}$ Recent genome-wide association studies have demonstrated that genetic factors are also involved in the development of OA. ${ }^{6}$ Meanwhile, some reports indicated a correlation between the polymorphism of some genes and the incidence of KOA. ${ }^{7,8}$

The incidence of obesity in Chinese significantly increased in the past decades. ${ }^{9}$ Obesity is a clinical manifestation characterized by an abnormal increase in the percentage of body fat to body weight due to the increase in the volume and number of adipocytes in the body, and excessive deposition of fat in some parts. Adipose tissue is an active endocrine organ that produces a wide spectrum of adipokines, ${ }^{10}$ which can regulate the functions of cartilage, synovium, bone and various immune cells in vivo, thus playing an important role in maintaining the balance of cartilage and bone growth. ${ }^{11}$ Recent studies ${ }^{3,12}$ have found that the infrapatellar fat pad, as a small adipose tissue, also secretes adipokines and pro-inflammatory mediators inducing synovial inflammation, and seems to act as an anatomo-functional unit with synovial membrane contributing to $\mathrm{OA}$ onset and progression. In view of the above interpretation, adipokines may be a key factor in the pathogenesis of $\mathrm{OA}$.

Omentin, produced and secreted primarily by visceral adipose tissues, is a newly discovered anti-inflammatory adipokine that is involved in disorders of blood vessels and metabolism and in a variety of chronic inflammatory diseases. ${ }^{13}$ It has two structural genes of omentin-1 and omentin-2. Of these, omentin-1 is the major form of omentin in the blood circulation. ${ }^{14}$ Previous research found that omentin-1 can participate in the pathogenesis of obesity and related diseases owing to an increased insulin sensitivity resulting from its stimulation on insulinmediated glucose uptake in human adipocytes. ${ }^{15} \mathrm{~A}$ recent study has verified that omentin-1 can eliminate the interleukin (IL)-1 $\beta$-induced degradation of type II collagen and aggrecan, the two main components of articular extracellular matrix, in a dose-dependent manner, ${ }^{16}$ highlighting a potential cartilage protection function of omentin- 1 .

Human omentin-1 gene is composed of eight exons and seven introns. Random sequencing showed that the single nucleotide missense mutation of Val109Asp is located in exon 4 of the omentin-1 gene. Published studies investigated the relationship of the Val109Asp polymorphism of the omentin- 1 gene with type 2 diabetes mellitus, chronic inflammatory bowel disease, psoriasis, nonalcoholic fatty liver disease, coronary artery disease, rheumatoid arthritis and nutritional behaviour regulation. ${ }^{17-23}$ Meanwhile, a recent study ${ }^{24}$ has discovered that the omentin-1 level in the peripheral blood and synovial fluid of patients with primary KOA is closely correlated with the severity of KOA and revealed that omentin-1 could be used as a potential biomarker for the degeneration of primary KOA. So far, the possible relationship of the genetic variability of the omentin-1 gene with KOA remains to be established.

On the above basis, the present study explored the relationship of the Val109Asp polymorphism of the omentin-1 gene with the risk of KOA. It also investigated the changes in omentin-1 levels in the peripheral blood and synovium fluid of the knee joint in patients with different genotypes of KOA and their relationship with the severity of KOA.

\section{Methods \\ Objects of Study}

The study included 383 Chinese Han patients (167 males and 216 females, aged 45-68 years old) with unilateral or bilateral primary KOA (KOA group) who were diagnosed and treated in the First Affiliated Hospital of Anhui Medical University from January 2018 to December 2019. All the enrolled patients with KOA met the criteria of the American Rheumatic Association, had typical symptoms and signs of KOA and X-ray signs of osteoarthritis in accordance with Kellgren-Lawrence (KL) Classification. ${ }^{25}$ The course of KOA was 3-16 years. Among the 383 patients with KOA, 186 cases received knee joint cavity arthrocentesis and obtained knee joint synovial fluid, and 78 cases were treated by knee arthroplasty. The major exclusion criteria were as follows: (i) patients with ankylosing spondylitis, psoriasis or other autoimmune diseases; (ii) patients with medical history of knee injury or intra-articular infection; (iii) patients with cardiovascular disease, chronic liver disease, diabetes, kidney disease, tumour and so on; (iv) patients with acute and chronic infectious diseases; (v) patients who received arthroscopic surgery of the knee in the past 6 months and steroid injection of the knee joint in the last 3 months and (vi) patients with obvious weight change in recent 3 months. In addition, 460 healthy individuals (192 males and 268 females, aged 47-67 years old) who received physical examination in the Health Management 
Center of our hospital during the same period were selected as the normal control group (NC group). All individuals in the $\mathrm{NC}$ group do not have joint pathology in the knee, which was confirmed by interrogation, physical examination and knee imaging examination (X-ray or MRI). In addition, they had no family history of OA, tumour, other chronic inflammation or chronic history. All participants were from the same race and region and no genetic relationship was found between them by genetic relationship investigation. The present study has been approved by the Medical Ethics Committee of the First Affiliated Hospital of Anhui Medical University, with informed consent obtained from all participants.

All participants in this study completed a questionnaire survey on lifestyle and personal disease history as described in a previous study. ${ }^{26}$ It covered the participants' smoking, drinking, occupation, sports activities, joint trauma and injury history, drugs (such as antihypertensive drugs, non-steroidal anti-inflammatory drugs (NSAIDs), etc.) and family history of OA. Furthermore, general information of enrolled participants were recorded, including body weight, height, waist circumference, hip circumference, systolic blood pressure (SBP) and diastolic blood pressure (DBP), associated with the calculation of body mass index (BMI) and waist hip ratio (WHR). The X-ray films of all patients with KOA were classified and evaluated based on the $\mathrm{K}-\mathrm{L}$ classification system. For bilateral KOA patients, the X-ray film of one side of the knee with more serious disease was selected for classification and evaluation. In accordance with the four imaging features of joint space narrowing, osteophyte, subchondral sclerosis and subchondral cyst, referring to previous studies, ${ }^{7} \mathrm{KOA}$ was further classified as mild-moderate (K-L: 1-2) or moderate-severe (K-L: 3-4).

\section{Laboratory Test}

After enrollment, all participants fasted for $10 \mathrm{~h}$ and took venous blood from their elbows to anticoagulant tubes or non-anticoagulant collection tubes in a fasting state from 8:00 to 8:30 the next morning. The collected blood samples after centrifugation were stored at $-80^{\circ} \mathrm{C}$ for subsequent analysis. Plasma samples were used to measure fasting plasma glucose (FPG), omentin-1, interleukin $1 \beta$ (IL-1 $\beta$ ) and interleukin 6 (IL-6). Meanwhile, serum samples were prepared to determine total cholesterol (TCH), triglyceride (TG), C-reactive protein (CRP) and other indicators. Blood glucose and blood lipid were measured using a fully automatic biochemical analyser (MODULE P800,
Roche, Switzerland). In specific, FPG was detected using the glucose oxidase method, TCH and TG through enzyme-linked colorimetry and CRP through latexenhanced immunoturbidimetric assay. In addition, the concentrations of omentin-1, IL-1 $\beta$ and IL-6 in the plasma and synovial fluid of the knee joint were determined by enzyme-linked immunosorbent assay (ELISA) in batches every 6 months in accordance with the instructions of this kit (Biovision Inc., USA) by Thermo Scientific ${ }^{\mathrm{TM}}$ Multiskan $^{\mathrm{TM}}$ FC Microplate Reader (Thermo Fisher Scientific, USA).

\section{DNA Extraction and Genotyping}

A $5 \mathrm{~mL}$ volume of venous blood was drawn from all participants to an ethylene diamine tetraacetic acid (EDTA) anticoagulant test tube. Genomic DNA was extracted using $5 \mathrm{~mL}$ whole blood samples using a DNA separation kit (Bioteke, Beijing, China). Genotype detection of the Val109Asp polymorphism of the omentin-1 gene was performed by detection of single nucleotide polymorphism (SNP). The forward primer was 5'GAGCCTTTAGGCCATGTCTCT-3' and the reverse primer was 5'-CTCTCCTTCTTCTCCAGCCCAT-3'. The primers mentioned above were synthesised by DNA Technology Company A/S (Takara Biotechnology (Dalian) Co., Ltd., China). PCR analysis was performed on Perform PCR analysis on PCR machine 310A (Hangzhou Longji Scientific Instrument Co., Ltd.) in accordance with the manufacturer's instructions. The final relative volume was $20 \mu \mathrm{L}$, including $2 \mu \mathrm{L}$ of primer, $1 \mu \mathrm{L}$ of template, $10 \mu \mathrm{L}$ of taq master mix and $7 \mu \mathrm{L}$ of $\mathrm{ddH} 2 \mathrm{O}$. The cycle conditions were described as follows: first, pre-denaturation at $94^{\circ} \mathrm{C}$ for $3 \mathrm{~min}$, denaturing at $94^{\circ} \mathrm{C}$ for $30 \mathrm{~s}$, annealing at $64^{\circ} \mathrm{C}$ for $30 \mathrm{~s}$, extension for $30 \mathrm{~s}$ at $72^{\circ} \mathrm{C}$ (14 cycles), then denaturing again at $94^{\circ} \mathrm{C}$ for $30 \mathrm{~s}$, annealing at $57^{\circ} \mathrm{C}$ for $30 \mathrm{~s}$, extension for $30 \mathrm{~s}$ at $72^{\circ} \mathrm{C}$, a final extension at $72^{\circ} \mathrm{C}$ for $10 \mathrm{~min}$. The PCR product was subjected to $2 \%$ agarose gel electrophoresis, and the target bands of the same size were cut out, and purified and recovered according to the magnetic bead method of the Yinglaidun recovery kit. Then use the Sanger sequencing experiment to measure the results, and use the $3730 \mathrm{xl}$ sequencer for sequencing. DNA Sequencing analysis software was used to analyze the sequencing results, Sequencing Analysis 5.2.0 software was used for interpretation, and Sequencher 5.1 software package was used for comparison analysis. 
A repeated test was conducted with about $5 \%$ samples randomly to verify the accuracy of genotyping results. The coincidence rate of all repeated samples was $100 \%$.

\section{Detection of IL-I $\beta$, IL-6, Matrix} Metalloproteinase I3 (MMP-I3) and Collagen-II (COL-II) in Articular Cartilage of Knee Joint

Under a sterile condition, the articular cartilage (about $10 \mathrm{~mm}$ in diameter and $8 \mathrm{~mm}$ in thickness) collected from 78 patients with KOA who underwent knee arthroplasty was sectioned, immediately placed into a mortar containing liquid nitrogen, ground into homogenate and then collected into $1.5 \mathrm{~mL}$ EP tubes. Each EP tube was added with $1 \mathrm{~mL}$ of RIPA high-intensity protein lysate (containing pepsin inhibitor, leucine inhibitor, trypsin inhibitor and phosphatase inhibitor), placed on ice for $10 \mathrm{~min}$ and then homogenised for $90 \mathrm{~min}$. After centrifugation at $12,000 \mathrm{r} / \mathrm{min}$ for $30 \mathrm{~min}$ at $4^{\circ} \mathrm{C}$, the supernatant was collected into EP tubes separately and stored at $-80^{\circ} \mathrm{C}$ for further detection. The contents of IL-1 $\beta$, IL-6, MMP13 and COL-II in the supernatant were determined by ELISA in batches every 6 months in accordance with the instruction of the kit (Biovision Inc., USA) by Thermo Scientific $^{\mathrm{TM}}$ Multiskan $^{\mathrm{TM}}$ FC Microplate Reader (Thermo Fisher Scientific, USA).

\section{Statistical Analysis}

SPSS 17.0 (SPSS, Chicago, IL, USA) was used for statistical analysis. Measurement data in accordance with normal distribution were expressed as mean \pm standard deviation $(\overline{\mathrm{x}} \pm \mathrm{s}), t$-test was used for comparison between the two groups, and one-way ANOVA was used for multigroup comparison, and LSD $t$-test for further pairwise comparison. Whereas non-normal data were expressed by median with interquartile range (IQR) $\left[\mathrm{M}\left(\mathrm{P}_{25}, \mathrm{P}_{75}\right)\right]$, non parametric $t$-test was used for comparison between two groups, Wilcoxon test was used for comparison among three groups, Bonferroni method was used to correct $\alpha$ $\left(\alpha^{\prime}\right)$, and Mann Whitney $U$-test was used for further pairwise comparison. The Chi-square test $\left(\chi^{2}\right)$ was used to evaluate the Hardy-Weinberg equilibrium. Difference of genotype and allele frequency between KOA patients and healthy controls was compared by $2 \times 2$ table and standard $\chi^{2}$ test. Odds ratios (ORs) and $95 \%$ confidence intervals (95\% CIs) were used to calculate the corresponding distribution of $\chi^{2}$ test. In addition, the correlation between
Val109Asp polymorphism and KOA was analysed by logistic regression analysis and a multiple regression model was established to control the effect of other confounding factors. Statistical significance was considered at a two-tailed $P$ value of less than 0.05 .

\section{Results}

\section{Characteristics of the Participants}

A total of 383 patients with KOA included 315 patients with bilateral knee lesions and 68 patients with unilateral knee lesions. No statistical differences were found in age, gender composition, smoking, drinking, physical exercise, SBP, DBP, BMI, WHR, FPG, TCH, TG and the levels of IL-1 $\beta$ and IL-6 in peripheral blood between the KOA and $\mathrm{NC}$ groups $(p>$ 0.05). Moreover, the proportion of NSAIDs and CRP level were significantly higher in the KOA group than in the $\mathrm{NC}$ group ( $p<0.05)$, but with an statistically reduced concentration of omentin-1 in plasma $[10.8(7.2,18.3)$ vs $19.2(10.8,28.7)$ $\mathrm{ng} / \mathrm{L}, p=0.028]$. In addition, the median duration of KOA symptoms was 42.5 months in this study, and $58.5 \%$ of patients were in grade 3-4 in accordance with K-L classification system. Detailed data are shown in Table 1.

\section{Correlation Between Vall09Asp Polymorphism of the Omentin-I Gene and Risk of KOA}

Agarose gel electrophoresis for PCR products of different genotypes of the omentin-1 gene and the representative results of different gene mutation types of the omentin-1 gene detected by SNP are shown in Figures 1 and 2, respectively. In $\mathrm{NC}$ group, the genotype distribution of the Val109Asp polymorphism of the omentin-1 gene was in accordance with the Hardy-Weinberg equilibrium $(p>0.0001)$, Whereas, this gene polymorphism did not conform to the Hardy-Weinberg equilibrium $(p<0.0001)$ in KOA group. The results are shown in Table S1. Multiple Logistic regression analysis revealed no significant correlation of the Val109Asp polymorphism of the omentin-1 gene with the incidence of KOA $(p>0.05)$. In addition, the frequency of Asp allele was lower in the KOA group (73.6\%) than in the NC group (76.7\%), but the difference was not statistically significant $(p>0.05)$ (Table 2).

The genotypes Val/Asp and Val/Val were combined for analysis. According to the diagnostic criteria for obesity in the Asia Pacific region issued by the World Health Organization ${ }^{27}$ and previous studies, ${ }^{7}$ a stratified analysis was carried out in the KOA and $\mathrm{NC}$ groups with a BMI of 
Table I Selected Characteristics of the Patients with KOA and Controls [( $\bar{x} \pm s), M(25 \%, 75 \%), n(\%)]$

\begin{tabular}{|c|c|c|c|}
\hline Characteristics & KOA $(n=383)$ & $N C(n=460)$ & $p$-value \\
\hline Age (year) & $51.3 \pm 12.1$ & $50.6 \pm 11.5$ & 0.312 \\
\hline \multicolumn{4}{|l|}{ Gender } \\
\hline Male & 167 & 192 & 0.683 \\
\hline Female & 216 & 268 & \\
\hline KOA symptoms & $42.5(18,68.3)$ & - & \\
\hline duration (months) & & & \\
\hline \multicolumn{4}{|l|}{ KOA location (\%) } \\
\hline Unilateral cases & $68(17.8)$ & - & \\
\hline Bilateral cases & $315(82.2)$ & - & \\
\hline \multicolumn{4}{|l|}{ Smokers (\%) } \\
\hline Current- & I0I (26.4) & $126(27.4)$ & 0.701 \\
\hline Ex- & $27(7.1)$ & $29(6.3)$ & 0.658 \\
\hline Alcohol (\%) & $78(20.4)$ & 91 (19.8) & 0.664 \\
\hline \multicolumn{4}{|l|}{ Physical exercise } \\
\hline \multicolumn{4}{|l|}{ (\%) } \\
\hline Never & $162(42.4)$ & $180(39.1)$ & 0.481 \\
\hline Occasional & $105(27.5)$ & II $3(24.6)$ & 0.372 \\
\hline Regular & $115(30.1)$ & $167(36.3)$ & 0.296 \\
\hline NSAIDs (\%) & $136(35.5)$ & $4(0.1)$ & $<0.001$ \\
\hline SBP (mmHg) & $|28 \pm| \mid$ & $127 \pm 10$ & 0.587 \\
\hline $\mathrm{DBP}(\mathrm{mmHg})$ & $82 \pm 10$ & $80 \pm 9$ & 0.321 \\
\hline BMI $\left(\mathrm{kg} / \mathrm{m}^{2}\right)$ & $24.34 \pm 3.44$ & $23.87 \pm 3.03$ & 0.384 \\
\hline WHR & $0.87 \pm 0.07$ & $0.86 \pm 0.06$ & 0.595 \\
\hline FPG (mmol/L) & $5.45 \pm 0.31$ & $4.97 \pm 0.21$ & 0.202 \\
\hline $\mathrm{TCH}(\mathrm{mmol} / \mathrm{L})$ & $4.69 \pm 0.69$ & $4.67 \pm 0.75$ & 0.503 \\
\hline TG (mmol/L) & $1.57 \pm 0.32$ & $1.5 I \pm 0.29$ & 0.492 \\
\hline \multicolumn{4}{|l|}{ K-L grade } \\
\hline I & 45 (II.7) & - & \\
\hline 2 & $114(29.8)$ & - & \\
\hline 3 & $142(37.1)$ & - & \\
\hline 4 & $82(2 I .4)$ & - & \\
\hline $\begin{array}{l}\text { Plasma IL-I } \beta \text { (pg/ } \\
\mathrm{mL})\end{array}$ & $0.7(0.5,1.4)$ & $0.6(0.3 .0 .9)$ & 0.285 \\
\hline Plasma IL-6 (pg/mL) & $20.3(15.3,26.8)$ & $15.2(8.5,21.1)$ & 0.078 \\
\hline Serum CRP (mg/L) & II.5 (8.9,I7.7) & $6.2(4.2,10.8)$ & 0.036 \\
\hline $\begin{array}{l}\text { Plasma omentin-I } \\
\text { (ng/L) }\end{array}$ & $10.8(7.2,18.3)$ & $\begin{array}{l}19.2(10.8 \\
28.7)\end{array}$ & 0.028 \\
\hline
\end{tabular}

Notes: Data are presented mean \pm standard deviations or numbers (\%) or median with interquartile range (IQR); Differences between two groups analyzed using the independent sample $t$-test to analyze the normally distributed continuous variables, using the non-parametric test to analyze the abnormally distributed continuous variables. And using $x^{2}$ test to analyze the count data.

Abbreviations: KOA, knee osteoarthritis; NC, normal control; NSAID, nonsteroid anti-inflammatory drug; SBP, systolic blood pressure; DBP, diastolic blood pressure; BMI, body mass index; WHR, weight hip ratio; FPG, fasting plasma glucose; TCH, total cholesterol; TG, triglyceride; KL, Kellgren-Lawrence; IL, interleukin; CRP, C-reaction protein.

$25 \mathrm{~kg} / \mathrm{m}^{2}$ and a cut-off age of 55 years old. As shown in Table 3, compared with Asp/Asp genotype carriers with BMI $<25 \mathrm{~kg} / \mathrm{m}^{2}$, obesity (BMI $\geq 25 \mathrm{~kg} / \mathrm{m}^{2}$ ) would increase the risk of developing KOA in Asp/Asp genotype carriers (adjusted OR $=2.564,95 \%$ CI 1.085-5.317, $\mathrm{p}=0.033$ ) and significantly increase the risk of KOA in Val109 allele carriers (adjusted OR $=1.416,95 \%$ CI 1.018-7.072, $p=0.042$ ). In addition, as listed in Table 4, compared with Asp/Asp genotype carriers aged $<55$ years old, increased age (age $\geq 55$ years old) would increase the risk of developing KOA in Asp/Asp genotype carriers (adjusted OR = $1.283,95 \%$ CI $1.492-9.186, p=0.046)$ and statistically increase the risk of KOA in Val109 allele carriers (adjusted $\mathrm{OR}=1.735,95 \%$ CI $1.107-8.681, p=0.038)$.

\section{Comparison of Clinical Parameters Among Different Genotypes in the KOA and NC Groups}

As shown in Table 5, no significant differences in mean age, gender composition, SBP, DBP, BMI, WHR, FPG, TCH, TG, IL-1 $\beta$, IL-6 and CRP levels were found among carriers with three genotypes in the KOA and $\mathrm{NC}$ groups, respectively $(p>0.05)$. In addition, no statistical differences in the proportion of patients with K-L grade 1 and K-L grade 2 as well as with K-L grade 3 and K-L grade 4 among carriers with three genotypes in the KOA group $(p>0.05)$. However, the Val109Asp polymorphism of the omentin-1 gene significantly influenced the plasma omentin-1 level in the KOA group. The plasma level of omentin-1 in Asp/Asp carriers was significantly higher than that in $\mathrm{Val} / \mathrm{Val}$ carriers $(p=$ 0.027), but no significant difference was found in the plasma level of omentin-1 between Asp/Asp and Val/Asp carriers as well as between Val/Asp and Val/Val carriers $(p>0.05)$. In the KOA group, the proportion of patients with moderatesevere $\mathrm{K}-\mathrm{L}$ grade in Asp/Asp carriers was significantly lower than that in Val/Val carriers $(p=0.039)$. Nevertheless, no statistical difference in the proportion of patients with moderate-severe K-L grade was found between Asp/Asp and Val/Asp carriers as well as between $\mathrm{Val} / \mathrm{Asp}$ and $\mathrm{Val} / \mathrm{Val}$ carriers $(p>0.05)$. In addition, no significant difference was found in plasma omentin-1 level among carriers with the three genotypes in the NC group ( $p>0.05)$.

\section{Comparison of the Levels of Omentin-I, IL-I $\beta$, IL-6 and CRP in Synovial Fluid and Expression of IL-I $\beta$, IL-6, MMP-I 3 and COL-II in Cartilage Tissue Among Different Genotypes in the KOA Group}

As listed in Table 6, the Val109Asp polymorphism of the omentin-1 gene in the KOA group exerted a significant impact on the levels of omentin-1, IL-1 $\beta$, IL- 6 and CRP in 


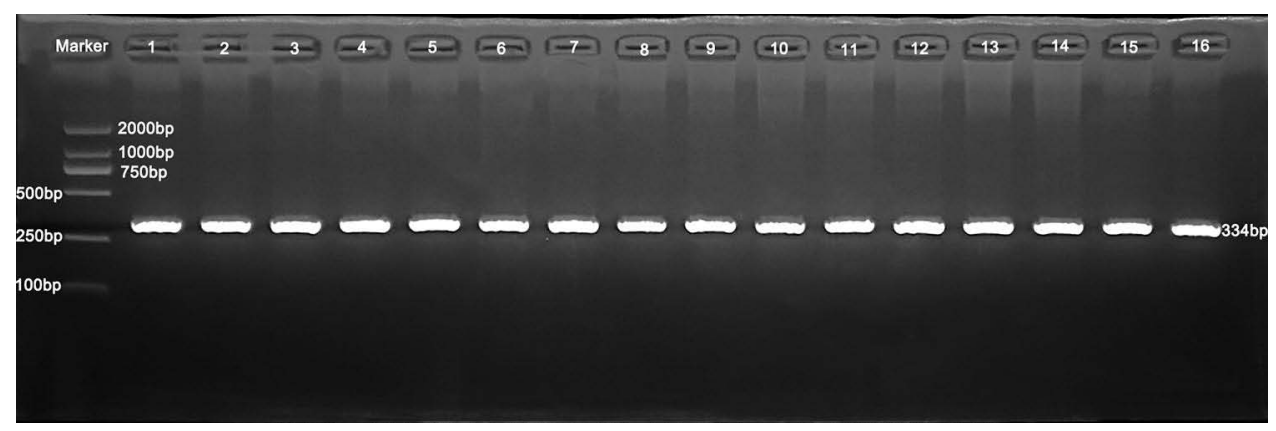

Figure I Agarose gel electrophoresis for PCR products of different genotypes of the omentin-I gene (334 bp). Val/Asp: Lane I, 3-6, 9, I2, I3, I6; Val/Val: Lane I0, II; Asp/ Asp: Lane 2, 7, 8, 14, 15

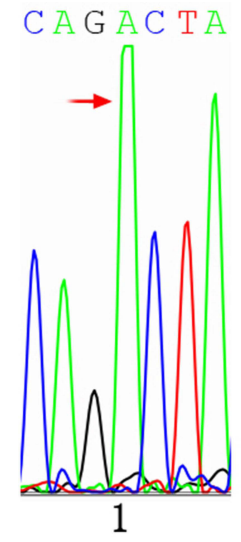

C A G T T A

G C A G T C T A
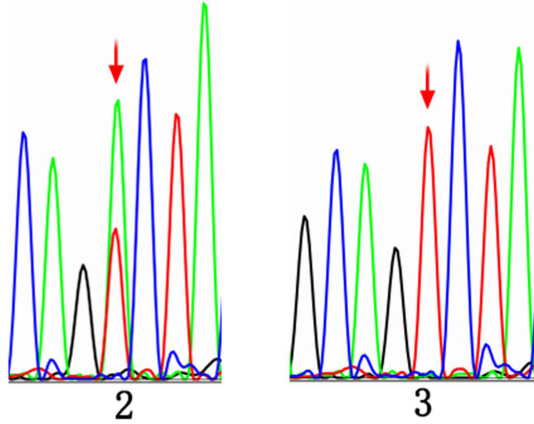

Figure 2 DNA sequence of omentin-I gene region showing omentin-I polymorphism in humans. DNA sequencing of the representative samples showing three genotypes (A/A, A/T and T/T) in omentin-I gene for Vall09Asp polymorphism. Among them, A/A genotype ( $\mathrm{I}$ ) is $\mathrm{Val} / \mathrm{Val}$ genotype, A/T genotype (2) is $\mathrm{Val} / \mathrm{Asp}$ genotype, T/T genotype (3) is Asp/Asp genotype. In the chromatogram Blue, Black, Green and Red peaks show C, G, A and T nucleotides respectively. The highlighted region in the chromatogram represents the site for Vall09Asp polymorphism. Sample I: GCAGCAAAGCAGA/ACTACCC. Sample 2: GCAGCAAAGCAGT/ ACTACCC. Sample 3: GCAGCAAAGCAGT/TCTACCC.

synovial fluid. Compared with Val/Val carriers, Asp/Asp carriers had statistically increased level of omentin-1 ( $p=$ 0.016 ), but with significantly decreased levels of IL-1 $\beta$,
IL-6 and CRP ( $p=0.017,0.024$ and 0.033 , respectively) in synovial fluid. In addition, no significant difference in the levels of omentin-1, IL-1 $\beta$, IL-6 and CRP in synovial fluid was found between Asp/Asp and Val/Asp carriers as well as between Val/Asp and Val/Val carriers $(p>0.05)$.

As presented in Table 7, the Val109Asp polymorphism of the omentin- 1 gene in the KOA group also affected the expression levels of IL-1 $\beta$, IL-6, MMP-13 and COL-II in cartilage tissue. In specific, the expression levels of IL-1 $\beta$, IL-6 and MMP-13 in cartilage tissue were significantly lower in Asp/Asp carriers than in Val/Val carriers ( $p=0.027,0.036$ and 0.032 , respectively), but with a highly increased expression level of COL-II ( $p=$ $0.025)$. Furthermore, no statistical difference in the expression levels of IL-1 $\beta$, IL-6, MMP-13 and COL-II in cartilage tissue was found between Asp/Asp and Val/Asp carriers as well as between Val/Asp and Val/Val carriers $(p>0.05)$.

\section{Discussion}

The findings of our study showed no significant difference in the Val109Asp polymorphism of the omentin-1 gene

Table 2 Logistc Regression Analysis of Association Between Omentin-I Gene Vall09Asp Polymorphism and Risk of KOA [n (\%)]

\begin{tabular}{|c|l|l|l|l|l|l|}
\hline Variables & KOA (n =383) & NC (n=460) & $\begin{array}{l}\text { Unadjusted Odds Ratios } \\
(\mathbf{9 5 \%} \mathbf{C l})\end{array}$ & p-value & Adjusted Odds Ratios (95\% CI) $^{\text {p-value }}$ \\
\hline $\begin{array}{c}\text { Genotypes } \\
\text { Asp/Asp }\end{array}$ & $226(59.0)$ & $282(61.3)$ & 1.000 (Reference) & - & 1.000 (Reference) \\
Val/Asp & $112(29.2)$ & $142(30.9)$ & $1.095(0.841-4.825)$ & 0.317 & $1.104(0.789-5.274)$ & - \\
Val/Val & $45(11.8)$ & $36(7.9)$ & $1.531(0.698-3.915)$ & 0.196 & $1.614(0.705-4.926)$ & 0.361 \\
Alleles & & & & & - & - \\
Asp & $564(73.6)$ & $706(76.7)$ & 1.000 (Reference) & - & - \\
Val & $202(26.4)$ & $214(23.3)$ & $1.136(0.695-6.124)$ & 0.282 & - \\
\hline
\end{tabular}

Note: ${ }^{a}$ Adjusted for age, gender, BMI, WHR, TCH, TG, lifestyle characteristics.

Abbreviations: KOA, knee osteoarthritis; NC, normal control; BMI, body mass index; WHR, weight hip ratio; TCH, total cholesterol; TG, triglyceride. 
Table 3 Multivariable Linear Regression Analysis of Association Between Omentin-I Gene Val I09Asp Polymorphism and Risk of KOA Stratified by BMI

\begin{tabular}{|l|l|l|l|l|l|l|l|}
\hline Genotypes & BMI $\mathbf{( k g / \mathbf { m } ^ { 2 } )}$ & $\begin{array}{l}\text { KOA } \\
\mathbf{( n = 3 8 3 )}\end{array}$ & $\begin{array}{l}\mathbf{N C} \\
\mathbf{( n = 4 6 0 )}\end{array}$ & $\begin{array}{l}\text { Unadjusted Odds Ratios } \\
\mathbf{( 9 5 \% ~ C l )}\end{array}$ & $\mathbf{p}$-value & $\begin{array}{l}\text { Adjusted Odds Ratios }_{\mathbf{( 9 5 \% ~ C l )}} \\
\text { p-value }\end{array}$ \\
\hline Asp/Asp & $<25$ & 71 & 137 & 1.000 (Reference) & - & 1.000 (Reference) & - \\
Val/Asp+Val/Val & $<25$ & 35 & 108 & $1.146(0.502-3.972)$ & 0.698 & $1.125(0.496-4.183)$ & 0.711 \\
Asp/Asp & $\geq 25$ & 175 & 125 & $2.952(1.264-6.104)$ & 0.029 & $2.564(1.085-5.317)$ & 0.033 \\
Val/Asp+Val/Val & $\geq 25$ & 102 & 90 & $1.583(1.097-7.314)$ & 0.037 & $1.416(1.018-7.072)$ & 0.042 \\
\hline
\end{tabular}

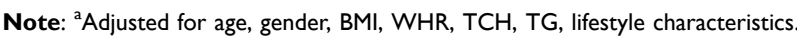

Abbreviations: KOA, knee osteoarthritis; NC, normal control; BMI, body mass index; WHR, weight hip ratio; TCH, total cholesterol; TG, triglyceride.

Table 4 Multivariable Linear Regression Analysis of Association Between Omentin-I Gene Val I09Asp Polymorphism and Risk of KOA Stratified by Age

\begin{tabular}{|c|c|c|c|c|c|c|c|}
\hline Genotypes & $\begin{array}{l}\text { Age } \\
\text { (Year) }\end{array}$ & $\begin{array}{l}\text { KOA } \\
(n=383)\end{array}$ & $\begin{array}{l}\text { NC } \\
(n=460)\end{array}$ & $\begin{array}{l}\text { Unadjusted Odds Ratios } \\
(95 \% \mathrm{Cl})\end{array}$ & $p$-value & $\begin{array}{l}\text { Adjusted Odds Ratios } \\
(95 \% \mathrm{Cl})\end{array}$ & $p$-value ${ }^{a}$ \\
\hline Asp/Asp & $<55$ & 52 & 153 & 1.000 (Reference) & - & I.000 (Reference) & - \\
\hline $\mathrm{Val} / \mathrm{Asp}+\mathrm{Val} / \mathrm{Val}$ & $<55$ & 49 & 89 & $1.089(0.683-2.529)$ & 0.326 & I.II7 (0.582-3.36I) & 0.338 \\
\hline Asp/Asp & $\geq 55$ & 194 & 109 & I.29I (I.754-8.562) & 0.042 & $1.283(1.492-9.186)$ & 0.046 \\
\hline $\mathrm{Val} / \mathrm{Asp}+\mathrm{Val} / \mathrm{Val}$ & $\geq 55$ & 88 & 109 & 1.862 (1.132-7.297) & 0.029 & $1.735(1.107-8.68 I)$ & 0.038 \\
\hline
\end{tabular}

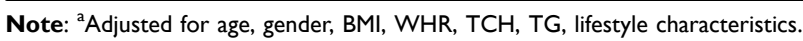

Abbreviations: KOA, knee osteoarthritis; NC, normal control; BMI, body mass index; WHR, weight hip ratio; TCH, total cholesterol; TG, triglyceride.

between the KOA and $\mathrm{NC}$ groups. Compared with Asp/ Asp genotype carriers with BMI $<25 \mathrm{~kg} / \mathrm{m}^{2}$ and age $<55$ years old, Val109 allele carriers with $\mathrm{BMI} \geq 25 \mathrm{~kg} / \mathrm{m}^{2}$ and age $\geq 55$ years old had significantly higher risk of KOA accordingly. These results suggest that the incidence of KOA may be increased owing to the interaction among BMI, age and Val109, although the Val109Asp polymorphism of the omentin-1 gene may not play a critical role in the pathogenesis of KOA in Chinese. In addition, our study for the first time reported that the Val109Asp polymorphism of the omentin-1 gene might lead to significant changes in the levels of omentin-1 in plasma and synovial fluid of the knee joint. Moreover, patients with Val/Val homozygote had more serious KOA, higher expression of inflammatory factors in synovial fluid and cartilage tissue and lower expression level of collagen. Collectively, the Val/Val genotype of the Val109Asp polymorphism of the omentin-1 gene may be a potential biomarker for the risk of KOA progression.

Several previous studies found that the level of omentin-1 in peripheral blood or synovial fluid is significantly correlated with the incidence of KOA. ${ }^{28-30}$ However, few studies focused on the correlation of the Val109Asp polymorphism of the omentin- 1 gene with the pathogenesis of KOA. Previous reports ${ }^{7,31}$ indicated that the single nucleotide polymorphisms of some genes are related to the occurrence of KOA, which may be useful biomarkers of KOA. In our study, the risk of KOA increased in Val/ Val genotype carriers $(\mathrm{OR}=1.614)$, but the difference was not statistically significant $(p=0.123)$. This result suggests no significant correlation between Val109Asp polymorphism and KOA in the Chinese Han population. However, after adjustment for the common possible risk factors of KOA, stratified analysis based on BMI and age revealed that the obese participants with Val109 allele and older people had a significantly increased risk of KOA (adjusted OR $=1.416$ and 1.735, $p=0.042$ and 0.038 , respectively). Accordingly, the interaction of Val109Asp polymorphism of omentin-1 gene with BMI and age may increase the risk of KOA in the Chinese Han population. Splichal et $\mathrm{al}^{23}$ revealed that the interaction of Val109 of the omentin-1 gene with BMI can reduce insulin sensitivity, which has a negative correlation with the occurrence of KOA. ${ }^{32}$ The above results strengthen our findings.

So far, experimental data on the effect of Val109Asp mutation on omentin-1 protein expression and function are lacking. In this study, no significant difference was found among different genotypes, although the omentin-1 level in the peripheral blood of Asp/Asp carriers was the highest 
Table 5 Comparison of Clinical Parameters of Different Genotypes in KOA Patients and Controls [( $\bar{x} \pm \mathrm{s}), \mathrm{M}(25 \%, 75 \%), \mathrm{n}(\%)]$

\begin{tabular}{|c|c|c|c|c|c|c|c|}
\hline \multirow{3}{*}{$\begin{array}{l}\text { Variables/Group } \\
\text { Age (year) }\end{array}$} & \multicolumn{3}{|l|}{ Genotypes } & \multirow[t]{2}{*}{ p-value } & \multicolumn{3}{|c|}{ Intergroup Comparisons ( $p$-value) } \\
\hline & \multirow[t]{2}{*}{ Val/Val } & \multirow[t]{2}{*}{ Val/Asp } & \multirow[t]{2}{*}{ Asp/Asp } & & & & \\
\hline & & & & & a & $\mathrm{b}$ & c \\
\hline KOA & $51.3 \pm 11.8$ & $51.1 \pm 12.4$ & $50.7 \pm 12.8$ & 0.832 & 0.794 & 0.801 & 0.838 \\
\hline NC & $50.6 \pm 10.9$ & $50.8 \pm 12.1$ & $49.9 \pm 11.2$ & 0.801 & 0.812 & 0.748 & 0.783 \\
\hline \multicolumn{8}{|c|}{ Gender (Male/Female), n (\%) } \\
\hline KOA & $14 / 18$ & $44 / 61$ & $109 / 137$ & 0.423 & 0.397 & 0.402 & 0.426 \\
\hline NC & $24 / 31$ & $59 / 80$ & $109 / 157$ & 0.395 & 0.402 & 0.328 & 0.371 \\
\hline \multicolumn{8}{|l|}{$\mathrm{SBP}(\mathrm{mmHg})$} \\
\hline KOA & $128 \pm 11$ & $129 \pm 11$ & $128 \pm 10$ & 0.794 & 0.763 & 0.692 & 0.801 \\
\hline NC & $127 \pm 10$ & $128 \pm 10$ & $127 \pm 1 \mid$ & 0.807 & 0.793 & 0.717 & 0.812 \\
\hline \multicolumn{8}{|l|}{$\mathrm{DBP}(\mathrm{mmHg})$} \\
\hline KOA & $82 \pm 10$ & $82 \pm 10$ & $83 \pm 11$ & 0.771 & 0.807 & 0.735 & 0.729 \\
\hline NC & $8 I \pm 9$ & $80 \pm 8$ & $80 \pm 9$ & 0.792 & 0.774 & 0.825 & 0.716 \\
\hline \multicolumn{8}{|l|}{ Body mass index $\left(\mathrm{kg} / \mathrm{m}^{2}\right)$} \\
\hline KOA & $23.98 \pm 3.82$ & $24.18 \pm 3.42$ & $24.47 \pm 3.38$ & 0.524 & 0.387 & 0.641 & 0.402 \\
\hline NC & $24.15 \pm 3.24$ & $23.95 \pm 3.15$ & $24.22 \pm 3.29$ & 0.498 & 0.506 & 0.411 & 0.528 \\
\hline \multicolumn{8}{|l|}{ Waist hip ratio } \\
\hline KOA & $0.87 \pm 0.07$ & $0.87 \pm 0.08$ & $0.88 \pm 0.07$ & 0.763 & 0.812 & 0.794 & 0.705 \\
\hline NC & $0.86 \pm 0.07$ & $0.86 \pm 0.07$ & $0.87 \pm 0.06$ & 0.797 & 0.824 & 0.756 & 0.785 \\
\hline \multicolumn{8}{|l|}{$\mathrm{FPG}(\mathrm{mmol} / \mathrm{L})$} \\
\hline KOA & $5.54 \pm 0.29$ & $5.5 I \pm 0.28$ & $5.52 \pm 0.31$ & 0.811 & 0.834 & 0.805 & 0.793 \\
\hline NC & $4.97 \pm 0.19$ & $5.1 I \pm 0.21$ & $4.98 \pm 0.23$ & 0.705 & 0.724 & 0.695 & 0.701 \\
\hline \multicolumn{8}{|c|}{ Total cholesterol (mmol/L) } \\
\hline KOA & $4.67 \pm 0.67$ & $4.68 \pm 0.71$ & $4.72 \pm 0.64$ & 0.684 & 0.598 & 0.612 & 0.675 \\
\hline NC & $4.64 \pm 0.75$ & $4.66 \pm 0.72$ & $4.68 \pm 0.78$ & 0.725 & 0.734 & 0.687 & 0.761 \\
\hline \multicolumn{8}{|l|}{ Triglyceride (mmol/L) } \\
\hline KOA & $1.56 \pm 0.29$ & $1.62 \pm 0.33$ & $|.56 \pm 0.3|$ & 0.637 & 0.607 & 0.598 & 0.642 \\
\hline NC & $|.52 \pm 0.3|$ & $1.49 \pm 0.28$ & $1.53 \pm 0.29$ & 0.504 & 0.461 & 0.487 & 0.526 \\
\hline \multicolumn{8}{|l|}{ Omentin-I (ng/L) } \\
\hline KOA & $9.7(7.2,11.3)$ & II.5 (8.8, 14.9) & I3.1 (9.8, 18.3) & 0.041 & 0.101 & 0.097 & 0.027 \\
\hline NC & $18.7(16.2,20.3)$ & $20.7(16.8,24.5)$ & $22.1(15.1,28.7)$ & 0.108 & 0.124 & 0.098 & 0.083 \\
\hline \multicolumn{8}{|l|}{ IL-I $\beta$ (pg/mL) } \\
\hline KOA & I.I $(0.8,1.5)$ & $0.9(0.7,1.3)$ & $0.8(0.6,1.2)$ & 0.187 & 0.161 & 0.212 & 0.155 \\
\hline NC & $0.5(0.3,0.8)$ & $0.6(0.4,0.9)$ & $0.5(0.3,0.8)$ & 0.412 & 0.394 & 0.402 & 0.525 \\
\hline \multicolumn{8}{|l|}{ IL-6 (pg/mL) } \\
\hline KOA & $23.1(18.3,26.5)$ & $20.6(19.8,27.1)$ & $18.2(15.8,23.4)$ & 0.097 & 0.143 & 0.107 & 0.075 \\
\hline NC & $17.9(\mid 2.5,21.7)$ & $15.3(10.8,19.2)$ & $13.6(8.5,17.5)$ & 0.086 & 0.073 & 0.176 & 0.085 \\
\hline \multicolumn{8}{|l|}{$\mathrm{CRP}(\mathrm{mg} / \mathrm{L})$} \\
\hline KOA & $15.3(11.4,18.1)$ & $13.7(10.4,16.3)$ & $11.3(8.7,15.1)$ & 0.105 & 0.127 & 0.261 & 0.097 \\
\hline NC & $6.8(4.9,9.8)$ & $5.7(3.1,10.1)$ & $4.9(2.9,7.8)$ & 0.113 & 0.149 & 0.202 & 0.084 \\
\hline $\mathrm{K}$-L grade $(\mathrm{I} / 2), \mathrm{n}(\%)$ & $3 / 5$ & $12 / 30$ & $30 / 79$ & 0.245 & 0.285 & 0.213 & 0.197 \\
\hline K-L grade (3/4), n (\%) & $14 / 10$ & $39 / 24$ & $89 / 48$ & 0.198 & 0.204 & 0.172 & 0.189 \\
\hline \multicolumn{8}{|c|}{ K-L grade $(3+4 / I+2), n(\%)$} \\
\hline KOA & $24 / 8$ & $63 / 42$ & $137 / 109$ & 0.046 & 0.087 & 0.112 & 0.039 \\
\hline NC & - & - & - & - & - & - & - \\
\hline
\end{tabular}

Notes: Data are presented mean \pm standard deviations or numbers (\%) or median with IQR; Differences among three groups analyzed using one-way ANOVA to analyze the normally distributed continuous variables, and LSD $t$ - test for further pairwise comparison. Whereas, for non-normal data, Wilcoxon test was used for comparison among three groups, Mann Whitney $U$-test was used for further pairwise comparison. And using $\chi^{2}$ test to analyze the count data. Intergroup comparisons: a (Val/Val vs Val/ Asp), b (Val/Asp vs Asp/Asp), c (Val/Val vs Asp/Asp).

Abbreviations: KOA, knee osteoarthritis; NC, normal control; SBP, systolic blood pressure; DBP, diastolic blood pressure; IL, interleukin; CRP, C-reaction protein; K-L, Kellgren-Lawrence. 
Table 6 Comparison of the Levels of Synovial Fluid Omentin- I, IL-I $\beta$, IL-6 and CRP Among Different Genotypes in KOA Patients [M $(25 \%, 75 \%)]$

\begin{tabular}{|l|l|l|l|l|l|l|l|l|}
\hline Variables & $\mathbf{n}$ & Val/Val & Val/Asp & Asp/Asp & P-value & \multicolumn{2}{|c|}{ Intergroup Comparisons (P-value) } \\
\cline { 5 - 8 } & & & & & & & a & b \\
\hline Omentin-I $(\mathrm{ng} / \mathrm{L})$ & 186 & $2.5(1.8,3.9)$ & $4.1(2.8,6.2)$ & $6.2(3.9,7.3)$ & 0.029 & 0.098 & 0.134 & 0.016 \\
IL-I $\beta(\mathrm{pg} / \mathrm{mL})$ & 186 & $6.3(3.9,8.7)$ & $4.4(2.5,6.6)$ & $2.6(1.2,5.1)$ & 0.023 & 0.114 & 0.087 & 0.017 \\
IL-6 $(\mathrm{pg} / \mathrm{mL})$ & 186 & $49.5(31.7,65.2)$ & $36.5(22.9,53.9)$ & $30.8(18.1,46.2)$ & 0.043 & 0.085 & 0.114 & 0.024 \\
CRP $(\mathrm{mg} / \mathrm{L})$ & 186 & $42.3(27.1,69.3)$ & $36.2(29.7,52.6)$ & $27.5(17.4,42.1)$ & 0.041 & 0.105 & 0.072 & 0.033 \\
\hline
\end{tabular}

Notes: Data are presented median with IQR; Wilcoxon test was used for comparison among three groups, Mann Whitney U-test was used for further pairwise comparison. Intergroup comparisons: a (Val/Val vs Val/Asp), b (Val/Asp vs Asp/Asp), c (Val/Val vs Asp/Asp).

Abbreviations: KOA, knee osteoarthritis; IL, interleukin; CRP, C-reaction protein.

Table 7 Comparison of the Expressions of IL-I $\beta$, IL-6, MMP-I3, Collagen-I and Collagen-III in Articular Cartilage of Knee Joint Among Different Genotypes in KOA Patients [M (25\%,75\%)]

\begin{tabular}{|l|l|l|l|l|l|l|l|l|}
\hline Variables & $\mathbf{n}$ & Val/Val & Val/ Asp & Asp/Asp & P-value & \multicolumn{2}{|l|}{ Intergroup Comparisons (P-value) } \\
\cline { 5 - 9 } & & & & & & a & b & c \\
\hline IL-I $(\mathrm{pg} / \mathrm{mL})$ & 78 & $15.2(7.9,22.1)$ & $12.7(8.4,19.2)$ & $9.6(4.2,12.4)$ & 0.033 & 0.102 & 0.098 & 0.027 \\
$\mathrm{IL}-6(\mathrm{pg} / \mathrm{mL})$ & 78 & $61.4(28.8,91.3)$ & $53.8(23.6,75.9)$ & $41.6(22.7,65.6)$ & 0.042 & 0.086 & 0.152 & 0.036 \\
MMP-I3 $(\mathrm{pg} / \mathrm{mL})$ & 78 & $118.3(27.7,55.2)$ & $92.8(48.3,134.6)$ & $78.1(31.6,103.4)$ & 0.039 & 0.075 & 0.119 & 0.032 \\
Collagen-II $(\mu \mathrm{g} / \mathrm{mL})$ & 78 & $61.9(28.7,107.1)$ & $80.5(39.3,123.7)$ & $101.9(56.8,212.1)$ & 0.031 & 0.143 & 0.096 & 0.025 \\
\hline
\end{tabular}

Notes: Data are presented median with IQR; Wilcoxon test was used for comparison among three groups, Mann Whitney U-test was used for further pairwise comparison. Intergroup comparisons: a (Val/Val vs Val/Asp), b (Val/Asp vs Asp/Asp), c (Val/Val vs Asp/Asp).

Abbreviations: KOA, knee osteoarthritis; IL, interleukin; MMP, Metalloproteinase.

in the $\mathrm{NC}$ group. In the KOA group, the levels of omentin1 in the peripheral blood and synovial fluid of Asp/Asp carriers were much higher than those of Val/Val carriers, which was independent of obesity. Splichal et $\mathrm{al}^{23}$ reported that the mean level of omentin- 1 in the peripheral blood of Asp/Asp homozygote carriers is $25 \%$ higher than that of $\mathrm{Val} / \mathrm{Val}$ homozygote carriers in the Central-European population, which supports the findings in our study. However, the causal relationship of the Val109Asp polymorphism of the omentin-1 gene with KOA cannot be determined based simply on the current results of this study. This result remains to be confirmed by a cohort study with a large sample size and further gene-protein functional analysis.

In view of the results of the present study, Asp/Asp genotype carriers and Val allele carriers in the NC group had similar age, gender, blood pressure, obesity status, blood lipid level and peripheral blood inflammatory factor levels. In the KOA group, compared with Asp/Asp genotype carriers, Val/Val genotype carriers had significantly higher expression levels of IL-1 $\beta$, IL- 6 and CRP in the synovial fluid of knee joint cavity as well as IL- $1 \beta$ and IL6 in the articular cartilage. Meanwhile, we observed an obvious increase in the expression of MMP-13, which can degrade cartilage matrix, but an evident decrease in the expression of COL-II, the main component in the articular cartilage matrix, resulting in a significantly serious degree of joint injury. Thus, KOA patients with Val/Val genotype may have a more "dangerous" phenotype. With regard to the above, findings of this study support that the Val109Asp polymorphism of the omentin-1 gene may be a promising biomarker to indicate the progression and severity of KOA.

Some studies have explored the value of omentin-1 in evaluating the illness condition of KOA. Li et $\mathrm{al}^{28}$ documented that KOA patients with a low level of omentin- 1 in synovial fluid may experience a more severe knee pain and joint disability (according to WOMAC score) compared with those with a high level of Omentin-1. Furthermore, some studies have confirmed a negative correlation of omentin-1 level in synovial fluid with the severity of KOA indicated by X-ray. ${ }^{28-30}$ All the aforementioned findings support the reliability of our research. However, additional studies also revealed no such clear correlation between the concentration of omentin-1 in synovial fluid and the severity of KOA. ${ }^{24}$ It may be attributed to the 
presence of ethnic differences of the enrolled subjects, the different inclusion criteria, the different analysis methods and so on in different studies. In addition, accumulated evidence has suggested that omentin-1 is a pleiotropic protective adipokine that provides a special repair function for articular chondrocytes. For instance, Li et al discovered that omentin-1 can inhibit the expression of MMP-1, MMP-3 and MMP-13 induced by proinflammatory factors by blocking the activation of the JAK-2/STAT3 signalling pathway and alleviate the degradation of the extracellular matrix protein of primary chondrocytes. ${ }^{33}$ Moreover, Chai et al verified in an in vitro study that omentin-1 can prevent IL-1 $\beta$-induced chondrocyte senescence by upregulating SIRT1 expression. ${ }^{16}$ So far, the protective mechanism of omentin-1 on chondrocytes remains to be further explored. Consistent with other studies, ${ }^{28}$ our study also found a significantly lower level of omentin-1 in the synovial fluid than in the peripheral blood of patients with KOA. Thus, the presence of other effector cells in patients with KOA may influence the expression of omentin-1 in knee joint cavity. Previous research ${ }^{34}$ revealed a complex network regulatory relationship between adipokines and OA development, which supports our view mentioned above.

It should be pointed out that there are several shortcomings in this study, mainly including the smaller sample size owing to the experimental design of a single-center study and the absence of exploration on other variants of the omentin-1 gene. Moreover, the distribution of Val109Asp polymorphism of the omentin-1 gene in KOA group did not conform to Hardy-Weinberg equilibrium. This imbalance may be caused by sampling error, sample size limitation and other confounding factors. Thus, additional studies related to this topic are needed. In addition, our study failed to clarify the causal relationship between Val109Asp polymorphism and KOA incidence. Future research is required to explore the effect of Val109Asp polymorphism on the function of omentin-1 protein and evaluate whether or not Val109Asp polymorphism can become a novel biomarker for evaluating the condition of $\mathrm{KOA}$.

\section{Conclusions}

The Val109Asp polymorphism of the omentin-1 gene may not be the main pathogenic factor of KOA in Chinese. The interaction among BMI, age and Val109 allele may increase the incidence of KOA. Val109 polymorphism may influence the levels of omentin-1 in the plasma and synovial fluid of patients with KOA and it is associated with the severity of KOA. The Val/Val genotype can be regarded as the potential biomarker for the risk of KOA progression. And further studies are need to evaluate the potential relationship between Val109Asp polymorphism of omentin-1 and incidence of KOA.

\section{Abbreviations}

OA, Osteoarthritis; KOA, Knee osteoarthritis; BMI, Body mass index; WHR, Waist hip ratio; NASIDs, Non-steroidal anti-inflammatory drugs; ELISA, Enzyme-linked immunosorbent assay; IL, Interleukin; MMP, Metalloproteinase; COL, Collagen; K-L, Kellgren-Lawrence; FPG, Fasting plasma glucose; TCH, Total cholesterol; TG, Triglyceride; CRP, C-reactive protein; EDTA, Ethylene diamine tetraacetic acid; PCR, Polymerase chain reaction; SNP, Single nucleotide polymorphism; SD, Standard deviation; IQR, Interquartile range.

\section{Data Sharing Statement}

The data sets generated during the study are available from the corresponding author on reasonable request.

\section{Ethics Approval}

All procedures performed in this study involving human participants have been approved by the Ethics Committee of the First Affiliated Hospital of Anhui Medical University and performed in accordance with the 1964 Helsinki Declaration and its later amendments or comparable ethical standards.

\section{Informed Consent}

Written informed consent was obtained from all the respondents.

\section{Acknowledgments}

We are grateful to the all patients for participating in the study. We thank the participants of this study including the doctors, nurses, and researchers from the Department of Rheumatology and Immunology, Department of Sports Injury and Arthroscopic Surgery, Department of Endocrinology and Health Management Center in the First Affiliated Hospital of Anhui Medical University.

\section{Author Contributions}

All authors made substantial contributions to conception and design, acquisition of data, or analysis and interpretation of data; took part in drafting the article or revising it 
critically for important intellectual content; agreed to submit to the current journal; gave final approval of the version to be published; and agreed to be accountable for all aspects of the work.

\section{Funding}

This study was supported by the Key Research and Development Projects of Anhui Province (Grant No. 1804h08020228) and the Natural Science Foundation of Anhui Province in China (2108085MH269). The funding body had no role in the design of the study, or the collection, analysis, and interpretation of data, or in writing the manuscript.

\section{Disclosure}

The authors declare that they have no competing interests.

\section{References}

1. Belluzzi E, Macchi V, Fontanella CG, et al. Infrapatellar fat pad gene expression and protein production in patients with and without osteoarthritis. Int $J$ Mol Sci. 2020;21(17):6016. doi:10.3390/ ijms21176016

2. Zhang L, Xing R, Huang Z, et al. Synovial fibrosis involvement in osteoarthritis. Front Med. 2021;8:684389. doi:10.3389/ fmed.2021.684389

3. Wang X, Hunter DJ, Jin X, Ding C. The importance of synovial inflammation in osteoarthritis: current evidence from imaging assessments and clinical trials. Osteoarthritis Cartilage. 2018;26 (2):165-174. doi:10.1016/j.joca.2017.11.015

4. Vina ER, Kwoh CK. Epidemiology of osteoarthritis: literature update. Curr Opin Rheumatol. 2018;30(2):160-167. doi:10.1097/ BOR.0000000000000479

5. Kulkarni K, Karssiens T, Kumar V, Pandit H. Obesity and osteoarthritis. Maturitas. 2016;89:22-28. doi:10.1016/j. maturitas.2016.04.006

6. Valdes AM, Spector TD. Genetic epidemiology of hip and knee osteoarthritis. Nat Rev Rheumatol. 2011;7(1):23-32. doi:10.1038/ nrrheum.2010.191

7. Shang H, Hao Y, Hu W, Hu X, Jin Q. Association between ADIPOQ gene variants and knee osteoarthritis in a Chinese population. Biosci Rep. 2019;39(3):BSR20182104. doi:10.1042/BSR20182104

8. Bai Y, Gao S, Liu Y, Jin S, Zhang H, Su K. Correlation between interleukin-17 gene polymorphism and osteoarthritis susceptibility in Han Chinese population. BMC Med Genet. 2019;20(1):20. doi:10.1186/s12881-018-0736-0

9. Wang Y, Xue H, Sun M, Zhu X, Zhao L, Yang Y. Prevention and control of obesity in China. Lancet Glob Health. 2019;7(9):e1166e1167. doi:10.1016/S2214-109X(19)30276-1

10. Scherer PE. Adipose tissue: from lipid storage compartment to endocrine organ. Diabetes. 2006;55(6):1537-1545. doi:10.2337/db060263

11. MacDonald IJ, Liu SC, Huang CC, Kuo SJ, Tsai CH, Tang CH. Associations between adipokines in arthritic disease and implications for obesity. Int $J$ Mol Sci. 2019;20(6):1505. doi:10.3390/ ijms20061505

12. Macchi V, Stocco E, Stecco C, et al. The infrapatellar fat pad and the synovial membrane: an anatomo-functional unit. J Anat. 2018;233 (2):146-154. doi:10.1111/joa.12820
13. Yang RZ, Lee MJ, Hu H, et al. Identification of omentin as a novel depot-specific adipokine in human adipose tissue: possible role in modulating insulin action. Am J Physiol Endocrinol Metab. 2006;290 (6):E1253-E1261. doi:10.1152/ajpendo.00572.2004

14. Shang FJ, Wang JP, Liu XT, et al. Serum omentin-1 levels are inversely associated with the presence and severity of coronary artery disease in patients with metabolic syndrome. Biomarkers. 2011;16 (8):657-662. doi:10.3109/1354750X.2011.622789

15. de Souza Batista CM, Yang RZ, Lee MJ, et al. Omentin plasma levels and gene expression are decreased in obesity. Diabetes. 2007;56 (6):1655-1661. doi:10.2337/db06-1506

16. Chai B, Zheng ZH, Liao X, et al. The protective role of omentin-1 in IL-1 $\beta$-induced chondrocyte senescence. Artif Cells Nanomed Biotechnol. 2020;48(1):8-14. doi:10.1080/21691401.2019.1699803

17. Schäffler A, Zeitoun $\mathrm{M}$, Wobser $\mathrm{H}$, Buechler C, Aslanidis C, Herfarth $\mathrm{H}$. Frequency and significance of the novel single nucleotide missense polymorphism Val109Asp in the human gene encoding omentin in caucasian patients with type 2 diabetes mellitus or chronic inflammatory bowel diseases. Cardiovasc Diabetol. 2007;6:3. doi:10.1186/1475-2840-6-3

18. Turan H, Yaykasli KO, Soguktas H, et al. Omentin serum levels and omentin gene Val109Asp polymorphism in patients with psoriasis. Int J Dermatol. 2014;53(5):601-605. doi:10.1111/ijd.12306

19. Yörük U, Yaykaşli KO, Özhan H, et al. Association of omentin Val109Asp polymorphism with coronary artery disease. Anadolu Kardiyol Derg. 2014;14(6):511-514. doi:10.5152/akd.2013.4932

20. Nazar S, Zehra S, Azhar A. Association of single nucleotide missence polymorphism Val109Asp of omentin-1 gene and coronary artery disease in Pakistani population: multicenter study. Pak J Med Sci. 2017;33(5):1128-1133. doi:10.12669/pjms.335.13110

21. Zhang C, Zhu KJ, Liu JL, et al. Omentin-1 plasma levels and omentin-1 expression are decreased in psoriatic lesions of psoriasis patients. Arch Dermatol Res. 2015;307(5):455-459. doi:10.1007/ s00403-015-1549-z

22. Kohan L, Safarpur M, Abdollahi H. Omentin-1 rs2274907 and resistin rs1862513 polymorphisms influence genetic susceptibility to nonalcoholic fatty liver disease. Mol Biol Res Commun. 2016;5(1):11-17.

23. Splichal Z, Bienertova-Vasku J, Novak J, et al. The common polymorphism Val109Asp in the omentin gene is associated with daily energy intake in the central-European population. Nutr Neurosci. 2015;18(1):41-48. doi:10.1179/1476830513Y.0000000100

24. Calvet J, Orellana C, Gratacós J, et al. Synovial fluid adipokines are associated with clinical severity in knee osteoarthritis: a cross-sectional study in female patients with joint effusion. Arthritis Res Ther. 2016;18(1):207. doi:10.1186/s13075-016-1103-1

25. Altman R, Asch E, Bloch D, et al. Development of criteria for the classification and reporting of osteoarthritis. Classification of osteoarthritis of the knee. Diagnostic and therapeutic criteria committee of the American Rheumatism Association. Arthritis Rheum. 1986;29 (8):1039-1049. doi:10.1002/art.1780290816

26. Chen MW, Ye S, Zhao LL, et al. Association of plasma total and high-molecular-weight adiponectin with risk of colorectal cancer: an observational study in Chinese male. Med Oncol. 2012;29 (5):3129-3135. doi:10.1007/s12032-012-0280-2

27. Yatsuya H, Li Y, Hilawe EH, et al. Global trend in overweight and obesity and its association with cardiovascular disease incidence. Circ J. 2014;78(12):2807-2818. doi:10.1253/circj.CJ-14-0850

28. Li ZG, Zhao DW, Xia CJ, et al. Decreased synovial fluid omentin-1 concentrations reflect symptomatic severity in patients with knee osteoarthritis. Scand J Clin Lab Invest. 2012;72(8):623-628. doi:10.3109/00365513.2012.726370

29. Xu L, Zhu GB, Wang L, Wang DF, Jiang XR. Synovial fluid omentin-1 levels are inversely correlated with radiographic severity of knee osteoarthritis. J Investig Med. 2012;60(3):583-586. doi:10.2310/JIM.0b013e31824443cb 
30. Gundogdu G, Gundogdu K, Miloglu FD, Tasc1 SY. A new perspective on the relation between obesity and knee osteoarthritis: omentin. Curr Rheumatol Rev. 2020;16(4):324-331. doi:10.2174/ 1573397116666191226122801

31. Shang H, Hao Y, Hu W, Hu X, Jin Q. OPN gene locus is associated with the risk of knee osteoarthritis: a case-control study. Biosci Rep. 2019;39(2):BSR20182023. doi:10.1042/BSR20182023

32. Ding L, Gao YH, Li YR, Huang YF, Wang XY, Qi X. Metabolic score for insulin resistance is correlated to adipokine disorder and inflammatory activity in female knee osteoarthritis patients in a Chinese population. Diabetes Metab Syndr Obes. 2020;13:2109-2118. doi:10.2147/DMSO.S249025
33. Li Z, Liu B, Zhao D, et al. Omentin-1 prevents cartilage matrix destruction by regulating matrix metalloproteinases. Biomed Pharmacother. 2017;92:265-269. doi:10.1016/j.biopha.2017.05.059

34. Carrión M, Frommer KW, Pérez-García S, Müller-Ladner U, Gomariz RP, Neumann E. The adipokine network in rheumatic joint diseases. Int J Mol Sci. 2019;20(17):4091. doi:10.3390/ijms20174091

\section{Publish your work in this journal}

Drug Design, Development and Therapy is an international, peerreviewed open-access journal that spans the spectrum of drug design and development through to clinical applications. Clinical outcomes, patient safety, and programs for the development and effective, safe, and sustained use of medicines are a feature of the journal, which has also been accepted for indexing on PubMed Central. The manuscript management system is completely online and includes a very quick and fair peer-review system, which is all easy to use. Visit http://www. dovepress.com/testimonials.php to read real quotes from published authors. 